\title{
Antibiotic prescription strategies and adverse outcome for uncomplicated lower respiratory tract infections: prospective cough complication cohort (3C) study
}

\author{
Paul Little, ${ }^{1}$ Beth Stuart, ${ }^{1}$ Sue Smith, ${ }^{2}$ Matthew J Thompson, ${ }^{3}$ Kyle Knox, ${ }^{2}$ Ann van den Bruel, ${ }^{2}$ \\ Mark Lown, ${ }^{1}$ Michael Moore, ${ }^{1}$ David Mant ${ }^{2}$
}

"University of Southampton, Primary Care Medical Group, PCPS Unit, Aldermoor Health Centre, Southampton S016 5ST, UK

2Nuffield Department of Primary Health Care Sciences, University of Oxford, Radcliffe Observatory

Quarter, Oxford, UK

3University of Washington, Seattle WA, USA

Correspondence to: P Little p.little@soton.ac.uk

Additional material is published online only. To view please visit the journal online.

Cite this as: $B M J$ J 2017;357:j2148 http://dx.doi.org/10.1136/bmj.j2148

Accepted: 24 April 2017

\section{ABSTRACT}

\section{OBJECTIVE}

To assess the impact on adverse outcomes of different antibiotic prescribing strategies for lower respiratory tract infections in people aged 16 years or more.

DESIGN

Prospective cohort study.

SETTING

UK general practice.

\section{PARTICIPANTS}

28883 patients with lower respiratory tract infection; symptoms, signs, and antibiotic prescribing strategies were recorded at the index consultation.

\section{MAIN OUTCOME MEASURES}

The main outcomes were reconsultation with symptoms of lower respiratory tract infection in the 30 days after the index consultation, hospital admission, or death. Multivariable analysis controlled for an extensive list of variables related to the propensity to prescribe antibiotics and for clustering by doctor.

RESULTS

Of the 28883 participants, 104 (0.4\%) were referred to hospital for radiographic investigation or admission, or both on the day of the index consultation, or were admitted with cancer. Of the remaining 28779 , subsequent hospital admission or death occurred in

\section{WHAT IS ALREADY KNOWN ON THIS TOPIC}

A Cochrane review identified trials showing modest impact of antibiotics on short term resolution of symptoms of lower respiratory tract infection but was unable to document major adverse outcomes

Two studies using the UK General Practice Research Database (GPRD) suggest that the risk of pneumonia might be reduced by antibiotic prescribing

Most pneumonias can be readily treated in community settings without hospital admission, but neither GPRD study recorded the impact on major adverse outcomes such as hospital admission or death, nor could they control for confounding by indication

\section{WHAT THIS STUDY ADDS}

After controlling for the clinical propensity to prescribe antibiotics, it was not possible to show that prescribing immediate or delayed antibiotics reduced the major adverse outcomes of subsequent hospital admission or death

Such events occur less than $1 \%$ of the time, and even using the most optimistic estimate of benefit, 400 patients would have to be treated to prevent one death or admission

Patients commonly experienced non-resolving or new symptoms, and if clinicians are considering antibiotics a delayed prescription may be preferable, since unlike immediate antibiotics it is likely to be associated with statistically significant $36 \%$ reduction in this outcome
26/7332 (0.3\%) after no antibiotic prescription, $156 / 17628(0.9 \%)$ after prescription for immediate antibiotics, and 14/3819 (0.4\%) after a prescription for delayed antibiotics. Multivariable analysis documented no reduction in hospital admission and death after immediate antibiotics (multivariable risk ratio $1.06,95 \%$ confidence interval 0.63 to 1.81 , $\mathrm{P}=0.84$ ) and a non-significant reduction with delayed antibiotics ( $0.81,0.41$ to $1.64, \mathrm{P}=0.61)$. Reconsultation for new, worsening, or non-resolving symptoms was common (1443/7332 (19.7\%), 4455/17 628 (25.3\%), and $538 / 3819$ (14.1\%), respectively) and was significantly reduced by delayed antibiotics (multivariable risk ratio $0.64,0.57$ to $0.72, \mathrm{P}<0.001$ ) but not by immediate antibiotics $(0.98,0.90$ to 1.07 , $\mathrm{P}=0.66)$.

\section{CONCLUSION}

Prescribing immediate antibiotics may not reduce subsequent hospital admission or death for young people and adults with uncomplicated lower respiratory tract infection, and such events are uncommon. If clinicians are considering antibiotics, a delayed prescription may be preferable since it is associated with a reduced number of reconsultations for worsening illness.

\section{Introduction}

Acute uncomplicated respiratory tract infections are the commonest acute illnesses managed in primary care in developed countries, and substantial numbers of patients still receive antibiotic treatment. ${ }^{1-3}$ The GRACE (Genomics to combat Resistance against Antibiotics for Community acquired lower respiratory tract infection (LRTI) in Europe) trial in lower respiratory tract infection ( $\mathrm{n}=2061)$ documented no clear benefit of antibiotics (hazard ratio for duration of "moderately bad" symptoms 1.06, 95\% confidence interval 0.96 to 1.18), ${ }^{4}$ and the updated Cochrane review provided similar estimates. ${ }^{5}$ Prescribing antibiotics incurs costs, increases reconsultation for subsequent episodes, medicalises self limiting illness, ${ }^{67}$ and promotes antibiotic resistance, which is dominated by prescribing of antibiotics in primary care. ${ }^{8}$ However, both clinicians and patients worry about more severe or prolonged illness and complications, ${ }^{9}$ and clinicians fear medicolegal consequences. ${ }^{210-12}$ Evidence to reassure clinicians is, however, limited: the Cochrane review did not document the impact of antibiotics on complications, and in the GRACE cohorts hospital admission or death occurred in fewer than $1 \%$ of patients who consulted, therefore large studies are needed. 
Trial data can be limited by external validity and substantially greater drug compliance compared with observational settings. ${ }^{1314}$ Thus observational studies provide important evidence to complement trial data, but conversely have the disadvantage of confounding by indication. Therefore the impact of antibiotics should be assessed using techniques to control for the propensity to prescribe..$^{15-17}$

We report the findings of an adequately powered observational study of lower respiratory tract infection in primary care using prospectively collected clinical data to assess the impact of antibiotics on adverse outcomes such as reconsultations with non-resolving or new symptoms, hospital admission, or death.

\section{Methods}

A structured proforma was used to document clinical presenting features and management at the index consultation. Review of medical records documented reconsultations with new or worsening illness, hospital admission, or death during the next 30 days.

The study took place in UK primary care. Participants were eligible if they were aged 16 years or more and had acute lower respiratory tract infection-defined as acute cough (new or worsening cough for three weeks or less) presenting as the main symptom and judged to be infective in origin by the doctor, consistent with the Cochrane review $^{18}$ and previous studies. ${ }^{4619-24}$ We excluded patients who had other causes of acute cough (eg, heart failure, acid reflux, fibrosing alveolitis), were unable to consent (eg, severe mental illness), were immunocompromised, had previously presented with the same episode of illness, or had been included previously.

\section{Data collection}

\section{Clinical record form}

Doctors completed a clinical record form in the consultation, documenting age, smoking history, duration of symptoms, nature of symptoms (dry or productive cough, shortness of breath, coryza, fever, chills or shivering, chest pain, headache, muscle aches, sleep disturbance, confusion, diarrhoea, sputum colour), examination results (respiratory rate, pulse, blood pressure, oxygen saturation, temperature, presence of wheeze, crepitations, or bronchial breathing), a rating of overall severity (visual analogue scale ranging from "well" to "very unwell"), and if antibiotics were prescribed.

\section{Outcomes (review of clinical records at 30 days)}

The main outcome measures were reconsultation in primary care or visit to an emergency department with progression of illness (ie, with non-resolution of symptoms or new symptoms or signs) $)^{25}$ in the 30 days after the index consultation, hospital admission, or death.

\section{Other data}

Comorbidities noted in the medical records were also documented. Lung comorbidity included asthma, chronic obstructive pulmonary disease, or history of other important lung disease requiring hospital investi- gation, and use of steroids or bronchodilators. Vaccination status (pneumococcal vaccination) was recorded.

Practice staff assessed the clinical records using a structured proforma. ${ }^{26}$ Most baseline data were captured by electronic data capture live during consultations, and the small proportion completed on paper was double entered. Data recorded on paper at follow-up review were double entered. The patient's postcode was used to assign deprivation, based on the national deprivation index.

\section{Sample size}

The target of 28024 patients was designed to detect predictors of rare (1:200) outcomes, with an odds ratio of 3 $(\alpha=0.01)$ and $80 \%$ power, among both those given antibiotics and those not given antibiotics. We also wanted to assess whether antibiotics reduced the number of uncommon outcomes: we assumed an antibiotic prescribing rate of $50 \%$ and estimated that the cohort would have more than $80 \%$ power to detect a one third reduction of outcome after antibiotics (odds ratio 0.66) for outcomes occurring with a frequency of $1 \%$ or more.

\section{Statistical analysis}

The primary analysis concerned those presenting initially with uncomplicated illness - that is, we excluded patients admitted to hospital on the day of the index consultation, admitted to hospital with cancer, and with pneumonia confirmed by radiography ordered on the day of the consultation. In the remaining cohort we assessed the impact of antibiotics using logistic regression, accounting for clustering by doctor and controlling for any potential confounder of the association between prescribing strategy and outcome. Levels of missing data were mostly low: $76 \%$ of participants had complete data and $17 \%$ were only missing data on oxygen saturation (see supplementary table). We assessed the possible change in the estimates if all the missing data were imputed with either all low values or all high values for oxygen saturation.

Covariates were dichotomised according to clinically meaningful cut-points. We assessed the impact of controlling for all potentially relevant clinical and demographic variables collected at first presentation and also generated a model when just controlling for significant covariates (from backward fitting of the regression model, retaining all variables with $\mathrm{P} \leq 0.20$ ). Relative risks were produced directly as the output from a generalised linear model for the binomial family. ${ }^{27}$ We then performed an analysis using a stratified propensity score method, ${ }^{15-17}$ which involved stratifying participants by fifths into mutually exclusive subsets based on their estimated propensity score, which in this dataset achieved a good balance of covariates in each stratum, key to providing control of confounding. This enabled exploration of whether more rigorous control for confounding by indication would modify the estimates. We calculated each propensity score comparing each prescribing strategy with no initial offer of antibiotics (ie, immediate antibiotics with no antibiotics, and delayed antibiotics with no antibiotics). 


\section{Patient involvement}

Since doctors' clinical behaviour was our key focus, working general practitioners were involved in the design of the clinical record form and in interpreting findings. Patients were involved in agreeing constituent studies of the overall programme; advising about the patient information leaflets, consent forms, clinical record form, and outcomes; and participating regularly in study management meetings.

\section{Results}

The National Institute for Health Research Clinical Research Network invited doctors nationwide in the UK to participate. Patients were recruited in 522 general practices between October 2009 and April 2013 after informed consent had been obtained (see supplementary figure). The practice median index of multiple deprivation score was 20.8, similar to the national figure of 21.5 from Public Health England. Overall, 28883 patients had baseline and follow-up data available and 28779 (99.6\%) had an initially uncomplicated illness at the index consultation. Of these, $7332(25.5 \%)$ received no prescription for antibiotics, 17628 (61.3\%) received a prescription for immediate antibiotics, and 3819 (13.3\%) received a prescription for delayed antibiotics (median advised delay 3 days, interquartile range 2-3 days) at the index consultation. There were no major differences in clinical characteristics between higher and lower recruiting practices (see appendix 1).

\section{Patient characteristics}

Major differences were found between antibiotic prescribing groups for some variables, particularly assessment of severity and lung findings. Participants prescribed immediate antibiotics were more likely to be older, have major comorbidities, and report more shortness of breath, fever, or purulent sputum, and the doctor was more likely to record higher severity ratings, low oxygen saturation, and crepitations or wheeze (table 1). Participants given a prescription for delayed antibiotics were commonly intermediate for the signs and symptoms between immediate and no prescription groups.

\section{Hospital admission or death after uncomplicated presentations}

Subsequent hospital admission or death occurred in 26/7332 (0.3\%) after no antibiotic prescription, $156 / 17628(0.9 \%)$ after an immediate prescription, and $14 / 3819(0.4 \%)$ after a delayed prescription. Respiratory infections accounted for the greatest number of hospital admissions ( $n=131)$ and deaths $(n=7)$, but cardiovascular or cerebrovascular events (admissions $n=19$ and

\begin{tabular}{|c|c|c|c|}
\hline Characteristics & No antibiotics & Immediate antibiotics & Delayed antibiotics \\
\hline Age $\geq 60$ years & 2112/7332 (28.8) & $7456 / 17628(42.3)$ & 1275/3819 (33.4) \\
\hline Female & $4448 / 7331(60.7)$ & $10334 / 17625(58.6)$ & $2284 / 3818(59.8)$ \\
\hline Illness duration $<7$ days & $3498 / 7332(47.7)$ & $8851 / 17628(50.2)$ & $1609 / 3819(42.1)$ \\
\hline Received pneumovax $<10$ years & $1031 / 7332(14.1)$ & $3642 / 17628(20.7)$ & $600 / 3819(15.7)$ \\
\hline Ever smoked & $3570 / 7227(49.4)$ & $9700 / 17327(56.0)$ & $1848 / 3757(49.2)$ \\
\hline Any comorbidity & 2696/7332 (36.8) & $8782 / 17628(49.8)$ & $1582 / 3819(41.4)$ \\
\hline Lung comorbidity & $1470 / 7332(20.1)$ & $5112 / 17628(29.0)$ & $855 / 3819(22.4)$ \\
\hline Taking steroids or bronchodilators & $1263 / 7057(17.9)$ & $4513 / 16767(26.9)$ & $735 / 3646(20.2)$ \\
\hline Living in top 10th deprivation area (most deprived)* & $1491 / 7332(20.3)$ & $3594 / 17628(20.4)$ & $654 / 3819(17.1)$ \\
\hline \multicolumn{4}{|l|}{ Symptoms: } \\
\hline Shortness of breath & $3874 / 7325(52.9)$ & $12306 / 17531(70.2)$ & $2268 / 3805(59.6)$ \\
\hline Fever & $2139 / 7329(29.2)$ & $7371 / 17570(42.0)$ & $1439 / 3813(37.7)$ \\
\hline Chills & $1824 / 7328(24.9)$ & $6116 / 17567(34.8)$ & $1183 / 3806(31.1)$ \\
\hline Chest pain & $2574 / 7325(35.1)$ & $6624 / 17574(37.7)$ & $1414 / 3809$ (37.1) \\
\hline Confusion & $399 / 7331(5.4)$ & $1241 / 17611(7.1)$ & $216 / 3819(5.7)$ \\
\hline Coryza & $4011 / 7325(54.8)$ & $9446 / 17552(53.8)$ & $2235 / 3805(58.7)$ \\
\hline Headache & $3127 / 7326(42.7)$ & $8254 / 17562(47.0)$ & $1841 / 3807(48.4)$ \\
\hline Muscle aches & $2364 / 7327(32.3)$ & $6747 / 17563(38.4)$ & $1360 / 3808(35.7)$ \\
\hline Diarrhoea & $563 / 7330(7.7)$ & $1600 / 17608(9.1)$ & $339 / 3816(8.9)$ \\
\hline Sputum: purulent & $3594 / 7329(49.0)$ & $12133 / 17628(68.8)$ & $2460 / 3818(64.4)$ \\
\hline Sputum: bloody/rusty & $159 / 7329(2.2)$ & $740 / 17628(4.2)$ & $110 / 3818(2.9)$ \\
\hline \multicolumn{4}{|l|}{ Clinical examination: } \\
\hline Severity assessment $\geq 5 / 10$ & $840 / 7332(11.5)$ & $9926 / 17628(56.3)$ & $1092 / 2819(28.6)$ \\
\hline Respiratory rate $>24 / \min$ & 440/7299 (6.0) & $2166 / 17557(12.3)$ & $264 / 3806(6.9)$ \\
\hline Temperature $\geq 37.8^{\circ} \mathrm{C}$ & $198 / 7328(2.7)$ & $1295 / 17612(7.4)$ & $150 / 3818(3.9)$ \\
\hline Pulse $\geq 100 / \mathrm{min}$ & $480 / 7329(6.6)$ & $2021 / 17620(11.5)$ & $287 / 3818(7.5)$ \\
\hline $\mathrm{O}_{2}$ saturation $<95 \%$ & $166 / 6180(2.7)$ & $1403 / 14350(9.8)$ & $120 / 3161(3.8)$ \\
\hline $\mathrm{SBP}<90 \mathrm{~mm} \mathrm{Hg}$ or DBP $<60 \mathrm{~mm} \mathrm{Hg}$ & $634 / 7332(8.7)$ & $1307 / 17628(7.4)$ & $247 / 3819(6.5)$ \\
\hline Crackles & $175 / 7330(2.4)$ & $11220 / 17622(63.7)$ & $814 / 3819(21.3)$ \\
\hline Bronchial breathing & $91 / 7329(1.2)$ & $1916 / 17618(10.9)$ & $149 / 3819(3.9)$ \\
\hline Wheeze & $585 / 7330(8.0)$ & $5908 / 17620(33.5)$ & $555 / 3819$ (14.5) \\
\hline
\end{tabular}

$\mathrm{SBP}=$ systolic blood pressure; $\mathrm{DBP}=$ diastolic blood pressure.

*Index of multiple deprivation: Office of National Statistics, 2007. 


\begin{tabular}{|c|c|c|c|c|c|c|c|}
\hline Factors & \multicolumn{2}{|c|}{ No antibiotics $(\mathrm{n}=7332)$} & \multicolumn{3}{|c|}{ Immediate antibiotics $(\mathrm{n}=17628)$} & \multicolumn{2}{|c|}{$\begin{array}{l}\text { Delayed antibiotics } \\
(\mathrm{n}=3819)\end{array}$} \\
\hline LRTI or URTI & $18(0.25)$ & $0(0.00)$ & $98(0.56)$ & $4(0.02)$ & $3(0.02)$ & $11(0.29)$ & $0(0.00)$ \\
\hline Cardiovascular or cerebrovascular event & $0(0.00)$ & $1(0.01)$ & $15(0.09)$ & $2(0.01)$ & $5(0.03)$ & $1(0.03)$ & $0(0.00)$ \\
\hline Cancer & $3(0.04)$ & $0(0.00)$ & $7(0.04)$ & $1(0.01)$ & $5(0.03)$ & $0(0.00)$ & $1(0.03)$ \\
\hline Other & $5(0.07)$ & $0(0.00)$ & $10(0.06)$ & $4(0.02)$ & $0(0.00)$ & $1(0.03)$ & $0(0.00)$ \\
\hline No reason given & $0(0.00)$ & $0(0.00)$ & $6(0.03)$ & $0(0.00)$ & $0(0.00)$ & $0(0.00)$ & $0(0.00)$ \\
\hline
\end{tabular}

deaths $n=8$ ) also occurred, in line with previous evidence of outcomes after lower respiratory tract infection, ${ }^{28}$ as did other infections $(n=12$ and $n=0)$ and a variety of other events such as collapse, dehydration, and acute renal failure ( $n=20$ and $n=4)$. Most hospital admissions were for respiratory infections: 18/26 (58\%) after no antibiotic prescription, 102/142 (72\%) after immediate antibiotics, and 11/14 (79\%) after delayed antibiotics (table 2). No admitted participant had allergic reactions, but one had amoxicillin induced cholestasis.

When accounting for the propensity to prescribe there was no reduction in hospital admission and death after immediate antibiotics (multivariable risk ratio $1.06,95 \%$ confidence interval 0.63 to $1.81, \mathrm{P}=0.84$ ) and a non-significant reduction with delayed antibiotics (0.81, 0.41 to $1.64, \mathrm{P}=0.61$; table 3$)$. For immediate antibiotics, adequate balance was obtained for covariates (see appendix 1), and for delayed prescribing the balance for most but not all covariates improved.

\section{Reconsultation with non-resolving or worsening symptoms}

Reconsultation for new, worsening, or non-resolving symptoms was common: 1443/7332 (19.7\%) for no antibiotics, 4455/17 628 (25.3\%) for immediate antibiotics, and 538/3819 (14.1\%) for delayed antibiotics. Delayed antibiotics were associated with a significant reduction in number of reconsultations (multivariable risk ratio $0.64,0.57$ to $0.72, \mathrm{P}<0.01$ ) but not immediate antibiotics (0.98, 0.90 to $1.07, \mathrm{P}=0.97$; table 4 ).

\section{Potential bias due to missing data on oxygen saturation}

The inferences were not changed either for reconsultations or for hospital admissions if missing values for oxygen saturation were imputed as either more than $95 \%$ saturation or less than $95 \%$ saturation (see supplementary table).

\section{Discussion}

As far as we are aware this is one of the very few prospective clinical cohorts with sufficient power to address the impact of antibiotic prescribing strategies on subsequent major adverse outcomes. Even in a cohort of 30000 participants, it was not possible to show that either immediate or delayed antibiotics were associated with a significant reduction in subsequent hospital admission or death. Non-resolving symptoms or progression of the illness with new symptoms was common, and a delayed prescription but not immediate antibiotics were associated with a significant reduction in reconsultations with such symptoms.

\section{Strengths and limitations of this study}

This study has several main strengths: 1) sufficient power to assess rare adverse outcomes owing to the size of the cohort; 2) high follow-up; 3) assessment of similar variables to previous trials and observational datasets $^{42229}$ and inclusion of the key drivers identified from qualitative work $^{30}$; 4) study of routine consultations in everyday primary care to provide a large generaliseable cohort, using a simple clinical proforma to facilitate recruitment; 5) a nationwide practice sample with deprivation scores similar to national figures; 6) the diagnosis of chest infections using clinical criteria similar to the Cochrane review ${ }^{18}$ and previous studies in

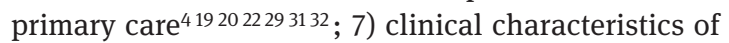
included participants similar to those in previous trials and observational cohorts in primary care c22 $23^{4}$ (approximately 20\% with lung comorbidity, 70\% with coloured sputum, previous illness duration of one week); 8) assessment of complications using a highly structured notes review for high reliability ${ }^{26}$; and 9) the rate of complications after presentation of a similar magnitude to previous studies using routine data. ${ }^{2}$

Limitations were that patients were recruited at the busiest times of year, and as with other studies of acute infection $^{1433}$ documentation of the details of those not approached was poor owing to time pressures (since time pressures to recruit also meant time pressure to document non-recruitment). No training was given in measuring clinical signs and we had no mechanism for quality assuring diagnostic skills, but conversely this means that these results are more generaliseable to the routine clinical setting. Although nearly $20 \%$ of participants had missing data for oxygen saturation, inferences were not altered in the sensitivity analysis with imputed extreme values for missing data. Patients were not blind to their management and so knowledge of the receipt of antibiotics might have altered their threshold for consulting. Using stratified propensity scores resulted in important changes in the estimates: for immediate antibiotics adequate balance of covariates 


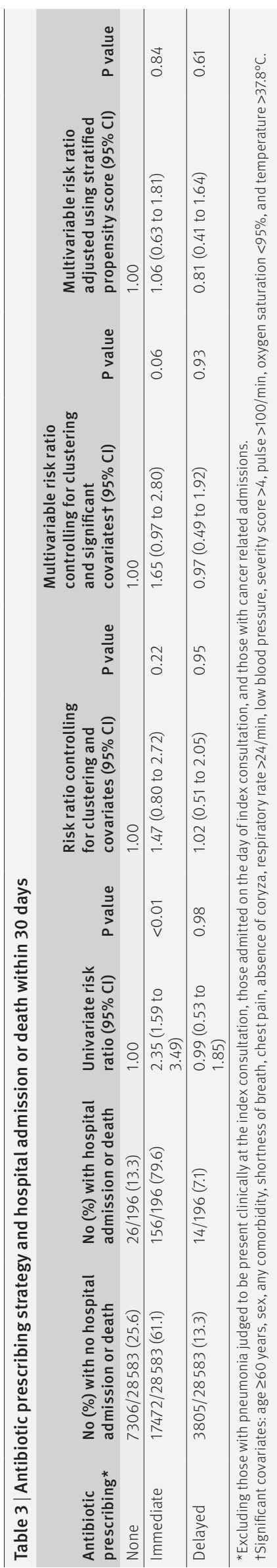

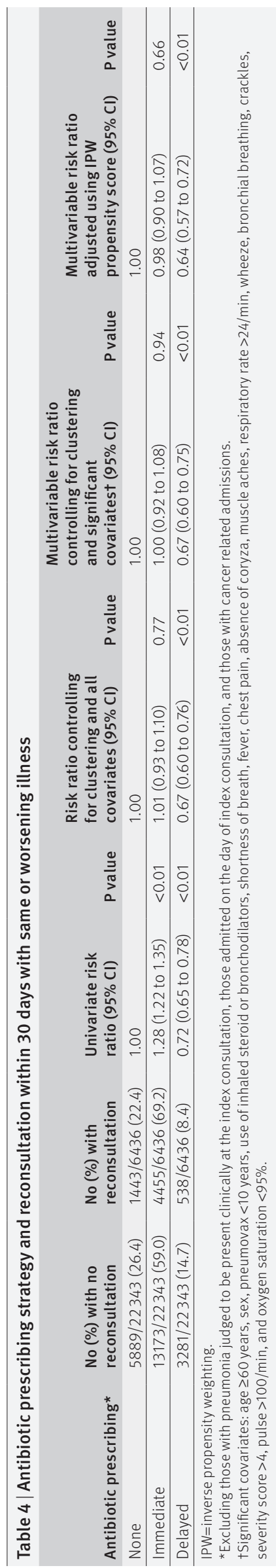

was achieved, making residual confounding less likely. For delayed prescribing the balance of covariates did not improve for all variables so we may have underestimated the impact of delayed prescribing on complications. We were also not powered to detect odds ratios greater than 0.66 for preventing complications. We did not measure reconsultations longer term, but there is reasonable trial evidence that both short and long term reattendance is reduced by delayed prescription. ${ }^{634}$

\section{Comparison with previous research}

We are aware of no large prospective cohort studies to have assessed the role of antibiotic prescribing strategies in everyday primary care practice for major adverse outcomes. Doctors are clearly using signs and symptoms to guide prescribing decisions: patients with more severe illness are more likely to receive a prescription for an antibiotic and those with intermediate severity delayed prescribing, which matches the behaviour of doctors in upper respiratory tract infections. ${ }^{26}$ The finding that subsequent hospital admission or death were not clearly reduced with delayed or immediate antibiotic prescribing could be due to inadequate control of confounding, but since we assessed a wide range of potentially confounding variables, a large impact of antibiotics seems unlikely. Even if we missed a small effect, subsequent major adverse events are uncommon, and in this cohort more than $60 \%$ of participants received an immediate antibiotic. If we use the lower limit of the $95 \%$ confidence interval of the risk ratio for immediate antibiotics ( 0.63 , ie, the most optimistic estimate of effect from this data) then approximately 400 people would still need to be treated to prevent one admission.

The major effect on reducing reconsultations for persistent, new, or worsening symptoms with delayed antibiotics almost mirrors the results of the large DESCARTE (Decision rule for the Symptoms and Complications of Acute Red Throat in Everyday practice) cohort in sore throat, which helps support the likely validity of the findings. ${ }^{26}$ Although the estimates are compatible with the large GRACE trial in lower respiratory tract infection, the DESCARTE study found that prescribing immediate antibiotics did slightly reduce the number of reconsultations, unlike the findings in the current cohort. The slight difference between these studies might relate to inadequate control of confounding by indication, or alternatively adherence: adherence is probably considerably worse for chest infections in routine settings ${ }^{13}$ than in the context of trials, ${ }^{4}$ hence the antibiotic dose and duration may have been suboptimal for participants in the current study, resulting in more prolonged symptoms. For delayed prescriptions the delay advised was much shorter than that in the trials, ${ }^{6}$ hence, as in the DESCARTE cohort, ${ }^{26}$ it is likely that more than half will have used their delayed prescription. It is unclear how delayed prescriptions work in reducing the number of reconsultations, but the delay allows more time for patients to decide whether symptoms have resolved or progressed, and the delayed start means that the course will also finish later when 
symptoms are more likely to be settling, and hence patients will perceive less need to revisit the doctor.

\section{Conclusion}

Prescribing immediate antibiotics may not reduce subsequent hospital admission or death for young people and adults with uncomplicated lower respiratory tract infection, and such events are uncommon. If clinicians are considering antibiotics, a delayed prescription may be preferable since it is associated with reduced number of reconsultations with worsening illness.

We thank Jan Charlwood for discussing and agreeing the main focus of the programme; Terry Hamer for early input into the protocols, commenting on patient materials, and suggesting key outcomes; Samantha Richards-Hall for commenting on materials and participating in regular management meetings for overseeing the programme; the local general practitioners who promoted the study; and the doctors, practices, and patients who participated.

Contributors: SS developed the protocol; provided day to day overall management of the study; coordinated recruitment, follow-up, and data entry; and commented on drafts of the paper. PL had the original idea for the protocol, led the funding application, supervised the running of the study, contributed to the analysis, led the drafting of the paper, and is the guarantor for the paper. DM had the original idea for the study, led the original development of the protocol for the funding application, led the further protocol development and ethics submission, provided overall supervision of the study, and contributed to the analysis and the drafting of the paper. MM developed the protocol for funding and contributed to the management of the study, developing the detailed plan for analysis, and the drafting of the paper. MJT, with SS and DM, led the development of the protocol for ethical approval, supervised the study, and contributed to the analysis and drafting of the paper. $\mathrm{KK}, \mathrm{AvdB}$, and $\mathrm{ML}$ helped develop the protocol and contributed to developing the detailed plan for analysis, the analysis, and the drafting of the paper. BS developed the analysis protocol and led the quantitative analysis with $\mathrm{DM}$ and $\mathrm{PL}$, and drafted the paper with $\mathrm{PL}$

Funding: This article presents independent research funded by the National Institute for Health Research (NIHR) under its programme grants for applied research programme (reference No RP-PG-040710098). The views expressed are those of the authors and not necessarily those of the NHS, the NIHR, or the Department of Health. The University of Southampton was the sponsor, but neither it nor the funder had any role in the running of the study, the analysis, the write-up, or the interpretation of the results.

Competing interests: All authors have completed the ICMJE uniform disclosure form at www.icmje.org/coi_disclosure.pdf (available on request from the corresponding author) and declare: no support from any other organisations (other than the NIHR service support detailed in the funding section) for the submitted work; no financial relationships with any organisations that might have an interest in the submitted work in the previous three years; no other relationships or activities that could appear to have influenced the submitted work.

Ethical approval: This study was approved by the Oxfordshire research ethics committee (A 09/H0604/67).

Data sharing: No additional data available.

Transparency: The manuscript's guarantor (PL) affirms that the manuscript is an honest, accurate, and transparent account of the study being reported; that no important aspects of the study have been omitted; and that any discrepancies from the study as planned (and, if relevant, registered) have been explained

This is an Open Access article distributed in accordance with the terms of the Creative Commons Attribution (CC BY 4.0) license, which permits others to distribute, remix, adapt and build upon this work, for commercial use, provided the original work is properly cited. See: http://creativecommons.org/licenses/by/4.0/.

1 Lee GC, Reveles KR, Attridge RT, et al. Outpatient antibiotic prescribing in the United States: 2000 to 2010. BMC Med 2014;12:96. doi:10.1186/1741-7015-12-96.

2 Petersen I, Johnson A, Islam A, Duckworth G, Livermore D, Hayward A. Protective effect of antibiotics against serious complications of common respiratory tract infections: retrospective cohort study with the UK General Practice Research Database. BMJ 2007;335:982. doi:10.1136/bmj.39345.405243.BE.
3 Gulliford M, Latinovic R, Charlton J, Little P, van Staa T, Ashworth M. Selective decrease in consultations and antibiotic prescribing for acute respiratory tract infections in UK primary care up to 2006. J Public Health (Oxf) 2009;31:512-20. doi:10.1093/pubmed/fdp081.

4 Little P, Stuart B, Moore M, et al. Amoxicillin for acute lower respiratory tract infection where pneumonia is not suspected clinically: a 12 country randomised placebo controlled trial in primary care. Lancet Infect Dis 2013;13:123-9. doi:10.1016/S1473-3099(12)70300-6.

5 Smith SM, Fahey T, Smucny J, Becker LA. Antibiotics for acute bronchitis. Cochrane Database Syst Rev 2014;(3):CD000245. doi:10.1002/14651858.CD000245.pub3.

6 Little P, Rumsby K, Kelly J, et al. Information leaflet and antibiotic prescribing strategies for acute lower respiratory tract infection: a randomized controlled trial. JAMA 2005;293:3029-35. doi:10.1001/ jama.293.24.3029.

7 Moore M, Little P, Rumsby K, et al. Effect of antibiotic prescribing strategies and an information leaflet on longer-term reconsultation for acute lower respiratory tract infection. Br J Gen Pract 2009;59:72834. doi:10.3399/bjgp09X472601.

8 Goossens H, Ferech M, Vander Stichele R, Elseviers M. ESAC Project Group. Outpatient antibiotic use in Europe and association with resistance: a cross-national database study. Lancet 2005;365:579-87. doi:10.1016/S0140-6736(05)70799-6.

9 Cornford CS. Why patients consult when they cough: a comparison of consulting and non-consulting patients. BrJ Gen Pract 1998;48:1751-4

10 Kumar S, Little P, Britten N.Why do GPs prescribe antibiotics for sore throat? A grounded theory interview study of general practitioners. BM/ 2003;326:138.

11 Little P, Watson L, Morgan S, Williamson I. Antibiotic prescribing and admissions with major suppurative complications of respiratory tract infections: a data linkage study. Br J Gen Pract 2002;52:187-90, 193.

12 Price D, Honeybourne D, Little P, et al. Recent trends in GP antibiotic prescribing practice: a potential link to increased community-acquired pneumonia mortality. Proceeding of the BTS conference. 2001;Dec 2001.

13 Francis NA, Gillespie D, Nuttall J, et al. GRACE Project Group. Antibiotics for acute cough: an international observational study of patient adherence in primary care. Br J Gen Pract 2012;62:e429-37. doi:10.3399/bjgp12X649124

14 Little P, Williamson I, Warner G, Gould C, Gantley M, Kinmonth AL. Open randomised trial of prescribing strategies in managing sore throat. BMJ 1997;314:722-7. doi:10.1136/bmj.314.7082.722.

15 Rosenbaum R, Rubin D. Reducing bias in observational studies using subclassification on the propensity score. I Am Stat AssoC 1984;79:516-24. doi:10.1080/01621459.1984.10478078.

16 Guo S, Fraser M. Propensity Score Analysis: Statistical Methods and Applications. 1st ed. Sage, 2010.

17 Deb S, Austin PC, Tu JV, et al. A Review of Propensity-Score Methods and Their Use in Cardiovascular Research. Can J Cardiol 2016;32: 259-65. doi:10.1016/j.cjca.2015.05.015.

18 Smith SM, Fahey T, Smucny J, Becker LA. Antibiotics for acute bronchitis. Cochrane Database Syst Rev 2014;(3):CD000245. doi:10.1002/14651858.CD000245.pub3.

19 MacFarlane J, Macfarlane RM, Rose D, Gard P, Holmes W. How common is pneumonia and other radiographic features in previously well adults who present in the community with acute lower respiratory tract illness? Eur Respir/1999;14:16s.

20 Macfarlane J, Holmes W, Gard P, et al. Prospective study of the incidence, aetiology and outcome of adult lower respiratory tract illness in the community. Thorax 2001;56:109-14. doi:10.1136/ thorax.56.2.109.

21 Butler JC, Hofmann J, Cetron MS, Elliott JA, Facklam RR, Breiman RF. The continued emergence of drug-resistant Streptococcus pneumoinia in the United states: an update from the centers for disease control and prevention Pneumococcal Sentinel Surveiilance System. J Infect Dis 1996;174:986-93. doi:10.1093/infdis/174.5.986.

22 Butler CC, Hood K, Verheij T, et al. Variation in antibiotic prescribing and its impact on recovery in patients with acute cough in primary care: prospective study in 13 countries. BMJ 2009;338:b2242. doi:10.1136/bmj.b2242.

23 van Vugt SF, Broekhuizen BD, Lammens C, et al. GRACE consortium. Use of serum $C$ reactive protein and procalcitonin concentrations in addition to symptoms and signs to predict pneumonia in patients presenting to primary care with acute cough: diagnostic study. BMJ 2013;346:f2450. doi:10.1136/bmj.f2450.

24 Moore M, Stuart B, Coenen S, et al. GRACE consortium. Amoxicillin for acute lower respiratory tract infection in primary care: subgroup analysis of potential high-risk groups. BrJ Gen Pract 2014;64:e75-80. doi:10.3399/bjgp14X677121.

25 Hay AD, Fahey T, Peters TJ, Wilson A. Predicting complications from acute cough in pre-school children in primary care: a prospective cohort study. Br/Gen Pract 2004:54:9-14.

26 Little P, Stuart B, Hobbs FD, et al. DESCARTE investigators. Antibiotic prescription strategies for acute sore throat: a prospective observational cohort study. Lancet Infect Dis 2014;14:213-9. doi:10.1016/S1473-3099(13)70294-9. 
27 Knol MJ, Le Cessie S, Algra A, Vandenbroucke JP, Groenwold RH. Overestimation of risk ratios by odds ratios in trials and cohort studies: alternatives to logistic regression. CMAJ 2012;184:895-9. doi:10.1503/cmaj.101715

28 Smeeth L, Thomas SL, Hall AJ, Hubbard R, Farrington P, Vallance P. Risk of myocardial infarction and stroke after acute infection or vaccination. N Engl/Med 2004:351:2611-8. doi:10.1056/NEIMoa041747.

29 Little P, Stuart B, Francis N, et al. GRACE consortium. Effects of internetbased training on antibiotic prescribing rates for acute respiratory-tract infections: a multinational, cluster, randomised, factorial, controlled trial. Lancet 2013;382:1175-82. doi:10.1016/S0140-6736(13)60994-0.

30 Brookes-Howell L, Hood K, Cooper L, et al. Clinical influences on antibiotic prescribing decisions for lower respiratory tract infection: nine country qualitative study of variation in care. BMJ Open 2012;2:e000795. doi:10.1136/bmjopen-2011-000795.

31 Holmes WF, Macfarlane JT, Macfarlane RM, Lewis S. The influence of antibiotics and other factors on reconsultation for acute lower respiratory tract illness in primary care. BrJ Gen Pract 1997;47:815-8.
32 Macfarlane J, Lewis SA, Macfarlane R, Holmes W. Contemporary use of antibiotics in 1089 adults presenting with acute lower respiratory tract illness in general practice in the U.K.: implications for developing management guidelines. Respir Med 1997;91:427-34. doi:10.1016/ S0954-6111(97)90258-4.

33 Little P, Gould C, Williamson I, Moore M, Warner G, Dunleavey J. Pragmatic randomised controlled trial of two prescribing strategies for childhood acute otitis media. BMJ 2001;322:336-42. doi:10.1136/ bmj.322.7282.336.

34 Little P, Gould C, Williamson I, Warner G, Gantley M, Kinmonth AL. Reattendance and complications in a randomised trial of prescribing strategies for sore throat: the medicalising effect of prescribing antibiotics. BM/1997;315:350-2. doi:10.1136/ bmj.315.7104.350.

Supplementary information: appendix 1 Supplementary figure: participant flow diagram 